\title{
Tumor Angiogenesis at Baseline Identified by 18F- Alfatide II PET/CT May Predict Survival Among Patients with Locally Advanced Non-Small Cell Lung Cancer Treated with Concurrent Chemoradiotherapy
}

\author{
Yuchun Wei \\ Shandong University \\ Xueting Qin \\ Shandong Cancer Hospital and Institute \\ Xiaoli Liu \\ Shandong University \\ Jinsong Zheng \\ Shandong Cancer Hospital and Institute \\ Xiaohui Luan \\ Dezhou People's Hospital
}

\section{Yue Zhou}

Shanghe People's Hospital

Jinming Yu

Shandong Cancer Hospital and Institute

Shuanghu Yuan ( $\nabla$ yuanshuanghu@sina.com )

Shandong Cancer Hospital: Shandong Cancer Hospital and Institute

\section{Research Article}

Keywords: 18F-alfatide, PET/CT, non-small cell lung cancer, chemoradiotherapy

Posted Date: September 3rd, 2021

DOI: https://doi.org/10.21203/rs.3.rs-729875/v1

License: (c) (i) This work is licensed under a Creative Commons Attribution 4.0 International License. Read Full License

Version of Record: A version of this preprint was published at Journal of Translational Medicine on February 2nd, 2022. See the published version at https://doi.org/10.1186/s12967-022-03256-3. 


\section{Abstract}

\section{Background}

The study investigated the predictive value of tumor angiogenesis observed by ${ }^{18} \mathrm{~F}$-ALF-NOTA-PRGD2 II (denoted as ${ }^{18} \mathrm{~F}$-Alfatide II) positron emission tomography (PET)/computed tomography (CT) before concurrent chemoradiotherapy (CCRT) for treatment response and survival among patients with locally advanced non-small cell lung cancer (LA-NSCLC).

\section{Methods}

Patients with unresectable stage IIIA or IIIB NSCLC (AJCC Cancer Staging 7th Edition) who received CCRT were included in this prospective study. All patients had underwent ${ }^{18} \mathrm{~F}$-Alfatide PET/CT scanning before CCRT, and analyzed parameters included maximum uptake values $\left(S_{U} V_{\text {max }}\right)$ of primary tumor $\left(S U V_{P}\right)$ and metastatic lymph nodes $\left(S U V_{L N}\right)$ and mean uptake value of blood pool ( $\left.S U V_{\text {blood }}\right)$. Tumor-to-background ratios (TBRS) were calculated. The ratios of $S U V_{P}$ to $S U V_{\text {blood }}, S U V_{L N}$ to $S U V_{\text {blood, }}$, and $S U V_{P}$ to $S U V_{L N}$ were denoted as $T B R_{P}, T B R_{L N}$, and T/LN. Short-term treatment response, progression-free survival (PFS), and overall survival (OS) were evaluated.

\section{Results}

Of 38 enrolled patients, 28 completed CCRT. SUV $, S_{P} S_{L N}, T_{B R}, T_{P R}$ and T/LN showed significant correlation with PFS (all $P<0.05)$. SUV $\mathrm{P}_{\mathrm{P}}$ was negatively correlated with OS $(P=0.005)$. SUV $\mathrm{P}_{\mathrm{P}}$ and $\mathrm{TBR}_{\mathrm{P}}$ were higher in non-responders than in responders $(6.55 \pm 2.74$ vs. $4.61 \pm 1.94, P=0.039 ; 10.49 \pm 7.58$ vs. $7.73 \pm 6.09, P=0.023$ ). Exploratory receiver operating characteristic curve analysis identified $\mathrm{TBR}_{\mathrm{P}}$ (area under the curve $[A U C]=0.764, P=0.018$ ), with a cutoff value of 6.52 , as the only parameter significantly predictive of the response to CCRT, with sensitivity, specificity, and accuracy values of $71.43 \%, 78.57 \%$, and $75.00 \%$, respectively. ROC curve analysis also identified $S U V_{P}(A U C=0.942, P<0.001$, cutoff value 4.64) and $\operatorname{TBR}_{\mathrm{P}}(\mathrm{AUC}=0.895, P=0.001$, cutoff value 4.95$)$ as predictive of OS with high sensitivity $(84.21 \%$, $93.75 \%)$, specificity $(100.00 \%, 66.67 \%)$, and accuracy $(89.29 \%, 82.14 \%)$.

\section{Conclusions}

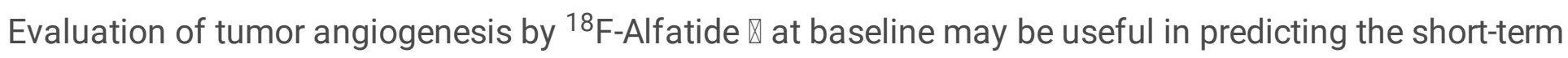
response to CCRT as well as PFS and OS in patients with LA-NSCLC.

\section{Background}

Locally advanced non-small cell lung cancer (LA-NSCLC) refers to stage III NSCLC, which is not suitable for treatment by surgical resection in the late stage of local foci [1]. These patients have regional lymph node invasion but no distant organ metastasis. Concurrent chemoradiotherapy (CCRT) is the standard treatment for LA-NSCLC [2, 3]. Variations in vascular networks can contribute to inter- and intratumor 
heterogeneity and regulate tumor growth, local invasiveness, and metastatic potential, ultimately influencing clinical outcomes [4]. Functional molecular imaging by positron emission tomography (PET) can be used to detect angiogenesis within a whole tumor.

The peptide arginine-glycine-aspartic acid peptide (Arg-Gly-Asp, commonly referred to as RGD) specifically binds to integrin av $\beta 3$, which is highly expressed in activated endothelial cells $[5,6]$. An ${ }^{18} \mathrm{~F}$ labeled PET imaging probe for RGD $\left({ }^{18} \mathrm{~F}\right.$-ALF-NOTA-PRGD2 II, denoted as ${ }^{18} \mathrm{~F}$-Alfatide II) represents a potentially feasible imaging tool for assessing tumor angiogenesis [7-9]. Studies of ${ }^{18} \mathrm{~F}$-Alfatide II have proven its safety and effectiveness for evaluating angiogenesis in NSCLC [10] with clarity and desirable image contrast, and phase III clinical trials are under way [11].

There are few reports on whether ${ }^{18} \mathrm{~F}$-Alfatide $\mathbb{Q}$ imaging of tumor angiogenesis offers predictive value for the long-term survival of NSCLC patients. In this study, we analyzed the standard uptake values (SUVs) of ${ }^{18} \mathrm{~F}$-Alfatide II on PET/computed tomography (CT) at baseline and explored the predictive value of these and related parameters for short-term treatment outcome, progression-free survival (PFS) and overall survival (OS) in LA-NSCLC patients treated with CCRT.

\section{Methods}

\section{Patients}

Between June 10, 2015, and Dec 28, 2016, a total of 38 patients with unresectable stage III NSCLC were enrolled in the study. This prospective study was approved by the local ethics committee of Shandong Cancer Hospital and Institute, and each patient gave written and informed consent before inclusion in the study. All patients were treated in Shandong Cancer Hospital and met the following criteria: (1) NSCLC diagnosed by histological and imaging examination such as $\mathrm{CT}$ or ${ }^{18} \mathrm{~F}$-fluorodeoxyglucose (FDG) PET/CT; (2) Eastern Cooperative Oncology Group (ECOG) score $\leq 1$; (3) clearly measurable metastatic lymph nodes and primary tumors (RECIST); and (4) age $>18$ years. All patients were ready to undergo ${ }^{18} \mathrm{~F}-$ Alfatide $\otimes \mathrm{PET} / \mathrm{CT}$ before chemotherapy or radiotherapy.

\section{Radiotracer preparation}

A simple lyophilized kit for labeling with PRGD2 peptide was purchased from the Jiangsu Institute of Nuclear Medicine, and the synthesis process was carried out as described in a previous study [12]. The radiochemical purity of the ${ }^{18} \mathrm{~F}$-alfatide $\otimes$ exceeded $99 \%$, and its specific radioactivity exceeded $37 \mathrm{GBq}$ $(1,000 \mathrm{mCi}) / \mu \mathrm{mol}$.

\section{PET/CT scanning}


Patients were given an intravenous injection of $4.81 \mathrm{MBq} / \mathrm{kg}(0.12 \mathrm{mCi} / \mathrm{kg}){ }^{18} \mathrm{~F}$-alfatide $\otimes$ and rested for about 60 minutes. Patients were not asked to fast or to confirm blood glucose levels. Scanning was performed with an integrated in-line PET/CT system (GEMINI TF Big Bore; Philips Healthcare). PET images were obtained from the head to the thigh, and the spiral CT component was performed with an Xray tube voltage peak of $120 \mathrm{kV}, 300 \mathrm{mAs}$. A full-ring dedicated PET scan of the same axial range followed. The patients continued normal shallow respiration during image acquisition. The images were attenuation-corrected with the transmission data from $\mathrm{CT}$. The attenuation-corrected PET images, CT images, and fused PET/CT images, displayed as coronal, sagittal, and trans axial slices, were viewed on a MEDEX workstation (Beijing, China).

\section{Image analysis}

Two experienced nuclear medicine physicians assessed the ${ }^{18} \mathrm{~F}$-alfatide $\triangle \mathrm{PET} / \mathrm{CT}$ images visually, referring to PET fusion and CT images, until consensuses were reached. Acquired ${ }^{18} \mathrm{~F}$-alfatide $\triangle \mathrm{PET} / \mathrm{CT}$ data were transferred to the workstation in the DICOM format. The radiotracer concentration in the region of interest (ROI) was normalized to the injected dose per kilogram of each patient's body weight to derive the standardized uptake values (SUVs). The SUVs were calculated according to the following formula: [measured activity concentration $(\mathrm{Bq} / \mathrm{ml}) \times$ body weight $(\mathrm{g})] /$ injected activity $(\mathrm{Bq})$.

PET/CT parameters, such as maximum SUV for primary tumor $\left(\mathrm{SUV}_{\mathrm{P}}\right)$ or metastatic lymph node $\left(\mathrm{SUV} \mathrm{V}_{\mathrm{LN}}\right)$, were generated using a vendor-provided automated contouring program. In addition, the maximal activity of $1 \mathrm{~cm}^{3}$ within the aortic arches was measured. Then the SUV ratios for primary tumor to aortic arches, metastatic lymph node to aortic arches, and primary tumor to metastatic lymph node were calculated, and denoted as $T B R_{P}, T B R_{L N}$, and $T / L N$, respectively.

\section{Chemoradiotherapy}

Patients were treated with two cycles of chemotherapy, followed by CCRT. Two additional cycles of chemotherapy were needed every 3 weeks after radiotherapy. An intensity-modulated radiotherapy technique (IMRT) or three-dimensional conformal RT (3D-CRT) was delivered to all patients with megavoltage equipment (6 MV). Radiotherapy was given as the conventionally fractionated regimen, 180-200 cGy for 5 days per week, and the total dose administered to patients ranged from 5040-6600 cGy (median dose, 6000 cGy). The chemotherapy administered was the cisplatin/docetaxel or cisplatin/pemetrexed regimen.

\section{Response evaluation and survival assessments}

Short-term outcome was assessed at 4 weeks after CCRT according to the revised RECIST criteria (v.1.1). According to the RECIST criteria, responders included patients with an outcome of complete response 
(CR) or partial response (PR), and patients who had an outcome of stable disease (SD) or progressive disease (PD) were classified as the non-responders.

PFS and OS were assessed for all NSCLC patients according to the RECIST criteria. OS and PFS were estimated from initiation of chemotherapy to death (for OS) and to progression or death (for PFS). Patients were followed up by enhanced CT every 6 weeks during treatment, every 2 months in the first year after treatment, and every 6 months from the second year after treatment.

\section{Statistical analysis}

Statistical analysis was performed using IBM SPSS Statistics for Windows version 20.0 (IBM, Armonk, $N Y$, USA). Quantitative data for $S U V_{P}, S U V_{L N}, T / L N, T B R_{P}$ and $T B R_{L N}$ were expressed as mean \pm standard deviation or median (range). Two-sample $t$ test and Wilcoxon rank-sum tests were used to compare the $\mathrm{PET} / \mathrm{CT}$ parameters between responders and non-responders. PET/CT parameters were tested by logistic regression analyses to identify the relationships between these variables and short-term outcomes or survival. Receiver-operating characteristic (ROC) curve analysis was used to determine the thresholds with the maximum Youden index as well as the predictive accuracy of ${ }^{18} \mathrm{~F}$-Alfatide II PET/CT parameters for treatment response and survival. Both PFS and OS were assessed by Kaplan-Meier analysis. All tests were two-sided, and $P<0.05$ was considered statistically significant.

\section{Result}

\section{Patient characteristics}

A total of 38 patients with pathologically proven stage III NSCLC were enrolled in the study, and of these, 28 completed CCRT and were included in the final analysis (Table 1). As of March 01, 2021, the median follow-up was 36.50 months (range, 9.00-72.50 months). The median PFS was 17 months $(95 \% \mathrm{Cl}$, 7.89 26.12 months), and the median OS was 36 months (95\% Cl, 11.24 60.76 months). The 1-, 3-, and 5 -year survival rates were $89.29 \%, 53.57 \%$ and $21.43 \%$, respectively.

\section{Correlations between PET parameters and survival}

The results of the correlation analyses for significant relationships between ${ }^{18} \mathrm{~F}$-Alfatide II PET/CT semiquantitative parameters and the PFS and OS of LA-NSCLC patients treated with CCRT are given in Table 2. SUV $V_{P}, S U V_{L N}, T B R_{P}, T B R_{L N}$ and T/LN were correlated significantly with PFS, and among them, $S U V_{P}$, $T_{B R}$ aN and T/LN were negatively correlated with PFS. Only SUV $V_{P}$ was negatively correlated with OS $(P=0.005)$. Statistically significant correlations were not observed for the other parameters. 


\section{Predictive value of PET parameters for short-term treatment outcome}

Several differences in SUV values were observed between responders and non-responders (Table 3 and Figure 2). SUV $V_{P}$ and $T_{B R}$ were significantly higher in non-responders than in responders $(6.55 \pm 2.74$ vs. $4.61 \pm 1.94, P=0.039 ; 10.49 \pm 7.58$ vs. $7.73 \pm 6.09, P=0.023)$. ROC curve analysis for the individual parameters, applying 'responder' as the dichotomous characteristic, revealed a significant area under the curve of $0.764(P=0.018)$ for $\mathrm{TBR}_{\mathrm{P}}$ (Figure 1, Table 4). With a cutoff value of 6.52 , derived from the Youden index, the sensitivity, specificity, and accuracy for $\mathrm{TBR}_{\mathrm{P}}$ were $71.43 \%, 78.57 \%$, and $75.00 \%$, respectively.

\section{Predictive value of PET parameters for survival}

ROC curve analysis was performed to determine the predictive accuracy of the ${ }^{18} \mathrm{~F}$-Alfatide II PET/CT parameters for survival of LA-NSCLC patients treated with CCRT. Highly significant correlations were observed between SUV and TBR and OS ( $P<0.001$ and $P=0.001$; Figure 3, Table 5). According to ROC curve analysis, the threshold values for $S U V_{P}, S U V_{L N}, T / L N, T B R_{P}$, and $T_{B R} R_{L N}$ were 4.64, 3.43, 1.73, 4.92, and 3.68, respectively (Table 6). The sensitivity, specificity, and accuracy of SUV $V_{P}$ for predicting OS were $84.21 \%, 100.00 \%$, and $89.29 \%$, respectively. The sensitivity, specificity, and accuracy of $\mathrm{TBR}_{\mathrm{P}}$ for predicting OS were $93.75 \%, 66.67 \%$, and $82.14 \%$, respectively.

\section{Discussion}

The results of this study indicate that for LA-NSCLC patients with CCRT, the baseline values of SUV $S_{S U V} V_{L N}, T_{B R}, T B R_{L N}$ and T/LN from pre-treatment ${ }^{18} F$-Alfatide II PET/CT imaging correlated significantly with PFS, and $S U V_{P}$ was negatively correlated with $O S$. TBR $R_{P}$ was an independent variable that may be useful for predicting short-term treatment outcome. From ROC curve analysis, $S_{U} V_{P}$ and $\mathrm{TBR}_{P}$ can predict OS with high sensitivity, specificity, and accuracy.

In clinical practice, physicians often observe differential treatment effects among different cancer patients with the same stage of disease, pathological type, and same treatment regimen [13]. The treatment of malignant tumors has entered the era of "precision medicine". Indeed, the heterogeneity of the tumor affects the therapeutic effect and prognosis [14]. The tissues for pathological examination and gene detection are all obtained through local sampling and biopsy, and thus, it is difficult to evaluate the overall metabolism of the tumor. As new tools for the accurate diagnosis of tumors as well as prognosis prediction, new imaging methods have promoted the rapid development of individualized accurate treatment of tumors. PET is a non-invasive modality for evaluating specific molecular features and a potential tool for the prediction of treatment response. Various molecular imaging techniques have been developed for predicting tumor response to therapy, such as ${ }^{18} \mathrm{~F}$-fluoro-D-glucose (FDG) PET [15], ${ }^{18} \mathrm{~F}$ - 
fluorothymidine (FLT) PET [16], ${ }^{18}$ F-fluroerythronitroimidazole (FETNIM) PET [17] and ${ }^{18} \mathrm{~F}-$ fluoromisonidazole (FMISO) PET [18].

Tumor angiogenesis as evaluated by ${ }^{18} \mathrm{~F}$-Alfatide II PET/CT before CCRT has been previously shown to predict the short-term treatment outcome in patients with advanced NSCLC [19], with the previous results showing that the maximum SUVs of tumor (SUVmax) and the SUVmax to SUVblood ratio (T/NTblood) were higher in non-responders $(P=0.0024, P=0.003)$. The corresponding AUCs determined by ROC curve analysis were $0.815(P=0.079)$ and $0.889(P=0.005)$, respectively. These results are consistent with the results of the present study, in which we instead analyzed pre-treatment baseline data from ${ }^{18} \mathrm{~F}$-Alfatide II PET/CT imaging and enrolled LA-NSCLC patients. Zhang et al [20] also reported the predictive sensitivity of ${ }^{18} \mathrm{~F}-\mathrm{RGD}$ imaging in glioblastoma patients treated with CCRT and found that both pre-treatment SUVs and intra-treatment SUVs were predictive parameters from ${ }^{18} \mathrm{~F}$-RGD PET/CT images. They also found that glioblastoma patients with higher SUVs were less likely to respond to CCRT. Li Li et al [21] reported that ${ }^{18} \mathrm{~F}-\mathrm{RGD}$ uptake on PET/CT imaging pre-treatment can predict the response to antiangiogenic therapy in patients who receive apatinib therapy. They enrolled patients with lung cancer, esophageal cancer, breast cancer, cervical cancer, ovarian cancer, stomach cancer, and other cancer types who were scheduled for second- or third-line apatinib therapy and found that higher SUVs on PET/CT predicted a better response to apatinib. From these findings, we can hypothesize that the addition of anti-angiogenesis therapy in cases with a poor response to radiotherapy and chemotherapy may improve the therapeutic effect.

In the present study, we provided the first clinical evidence that ${ }^{18} \mathrm{~F}$-Alfatide II uptake parameters at baseline are predictive of survival in patients with LA-NSCLC treated with CCRT. The tumor vascular network is dynamic and can limit tumor growth [22-24] by mediating the transportation of chemotherapeutic drugs and affecting the sensitivity to radiotherapy [25]. Microvessel density has been reported to be a significant prognostic factor for poor outcome in NSCLC [26]. Integrin av $\beta 3$, which forms complexes with vascular endothelial growth factors, is the most important integrin in the process of angiogenesis and is highly expressed on newly formed vessels $[27,28]$. In several studies, the accuracy of RGD PET imaging for evaluating the tumor vasculature via imaging of integrin av $\beta 3$ was verified [29-31]. This supports our finding that ${ }^{18} \mathrm{~F}$-Alfatide II PET/CT can be useful for predicting the survival of NSCLC patients.

This study has several limitations in addition to the relatively small sample sizes. This was a singlecenter study. In this study, ${ }^{18}$ F-Alfatide $\triangle \mathrm{PET} / \mathrm{CT}$ imaging was performed only once in NSCLC patients before treatment, and not during or after treatment. Further research to determine whether changes in the SUVs on ${ }^{18} \mathrm{~F}$-Alfatide II PET/CT correlate with prognosis is warranted. Nevertheless, these shortcomings diminish neither the potential of our findings nor the importance of dedicated prospective investigations to corroborate these findings.

\section{Conclusions}


The results of this prospectively study confirm that pre-treatment tumor angiogenesis as evaluated by

${ }^{18} \mathrm{~F}$-Alfatide II PET/CT imaging may be useful in predicting the short-term outcome of CCRT as well as the PFS and OS in patients with advanced NSCLC. For LA-NSCLC patients with higher SUVs on baseline ${ }^{18} \mathrm{~F}$ Alfatide II PET/CT images, the addition of anti-vascular therapy may improve the efficacy of CCRT and prolong survival.

\section{Abbreviations}

${ }^{18} \mathrm{~F}$-Alfatide, ${ }^{18} \mathrm{~F}$-ALF-NOTA-PRGD2; PET/CT, positron emission tomography / computed tomography; CCRT, concurrent chemoradiotherapy; LA-NSCLC, locally advanced non-small cell lung cancer; SUV $_{\text {max, }}$ maximum uptake values; $S U V_{P}$, maximum uptake values of primary tumor; $S U V_{L N}$, maximum uptake values of metastatic lymph nodes; $S U V_{\text {blood }}$, mean uptake value of blood pool; TBRs, tumor-tobackground ratios; $T B R_{P}$, ratios of $S U V_{P}$ to $S U V_{\text {blood }}, T B R_{L N}$, ratios of $S U V_{L N}$ to $S U V_{\text {blood }} T / L N$, ratios of SUV $_{\mathrm{P}}$ to $\mathrm{SUV}_{\mathrm{LN}}$; PFS, progression-free survival, OS, overall survival; $C R$, complete response; PR, partial response, $\mathrm{SD}$, stable disease; $\mathrm{PD}$, progressive disease.

\section{Declarations}

\section{Ethics approval and consent to participate:}

This prospective study was approved by the local ethics committee of Shandong Cancer Hospital and Institute, and each patient gave written and informed consent before inclusion in the study.

\section{Consent for publication:}

All the personal data involved in this article have been signed with informed consent.

\section{Availability of data and materials:}

The datasets used and/or analysed during the current study are available from the corresponding author on reasonable request.

\section{Competing interests:}

The authors declare that they have no competing interests.

\section{Funding:}


The study was supported by funds from the Major Scientific and Technological Innovation Projects of Shandong (2018YFJH0502), the Academic Promotion Program of Shandong First Medical University (2019ZL002) and the foundation of National Natural Science Foundation of China $(81872475,81372413$, 81627901 and 82030082).

\section{Authors' contributions:}

Shuanghu Yuan and Jinming Yu conceived of the study and participated in its designed. Yuchun Wei participated in the experiments and drafted the manuscript. Qinxue Ting, Xiaohui Luan and Yue Zhou contributed to the sample collection and interpretation the data. Xiaohui Luan performed the statistical analysis. Jinsong Zheng carried out the nuclear medicine. All authors read and approved the final manuscript.

\section{Acknowledgments:}

The authors thank Laney Webber for English language editing services.

\section{References}

1. Aupérin A, Le PC, Rolland E, Curran WJ, Furuse K, Fournel P, et al. Meta-analysis of concomitant versus sequential radiochemotherapy in locally advanced non-small-cell lung cancer. J Clin Oncol. 2010;28(13):2181-90.

2. Pignon JP, Stewart LA. Randomized trials of radiotherapy alone versus combined chemotherapy and radiotherapy in stages IIla and IIlb nonsmall cell lung cancer: a meta-analysis. Cancer. 1996;77(11):2413-4.

3. Marino P, Preatoni A, Cantoni A. Randomized trials of radiotherapy alone versus combined chemotherapy and radiotherapy in stages IIla and IIlb nonsmall cell lung cancer. A meta-analysis. Cancer. 1995;76(4):593-601.

4. Pritchard RS, Anthony SP. Chemotherapy plus radiotherapy compared with radiotherapy alone in the treatment of locally advanced, unresectable, non-small-cell lung cancer. A meta-analysis. Ann Intern Med. 1996;125(9):723-9.

5. Hood JD, Cheresh DA. Role of integrins in cell invasion and migration. Nat Rev Cancer. 2002;2(2):91100.

6. Liu Z, Wang F, Chen X. Integrin alpha(v)beta(3)-Targeted Cancer Therapy. Drug Dev Res. 2008;69(6):329-39.

7. Kumar CC. Integrin alpha $v$ beta 3 as a therapeutic target for blocking tumor-induced angiogenesis. Curr Drug Targets. 2003;4(2):123-31. 
8. Yu YP, Wang Q, Liu YC, Xie Y. Molecular basis for the targeted binding of RGD-containing peptide to integrin aVß3. Biomaterials. 2014;35(5):1667-75.

9. lagaru A, Gambhir SS. Imaging tumor angiogenesis: the road to clinical utility. AJR Am J Roentgenol. 2013;201(2):W183-91.

10. Gao S, Wu H, Li W, Zhao S, Teng X, Lu H, et al. A pilot study imaging integrin av $\beta 3$ with RGD PET/CT in suspected lung cancer patients. Eur J Nucl Med Mol Imaging. 2015;42(13):2029-37.

11. Wu C, Yue X, Lang L, Kiesewetter DO, Li F, Zhu Z, et al. Longitudinal PET imaging of muscular inflammation using 18F-DPA-714 and 18F-Alfatide II and differentiation with tumors. Theranostics. 2014;4(5):546-55.

12. Wan W, Guo N, Pan D, Yu C, Weng Y, Luo S, et al. First experience of 18F-alfatide in lung cancer patients using a new lyophilized kit for rapid radiofluorination. J Nucl Med. 2013;54(5):691-8.

13. Nakajima N, Kataoka M, Sugawara $Y$, Ochi T, Kiyoto S, Ohsumi S, et al. Volume-based parameters of 18F-fluorodeoxyglucose positron emission tomography/computed tomography improve disease recurrence prediction in postmastectomy breast cancer patients with 1 to 3 positive axillary lymph nodes. Int J Radiat Oncol Biol Phys. 2013;87(4):738-46.

14. Wolf Y, Bartok O, Patkar S, Eli GB, Cohen S, Litchfield K, et al. UVB-Induced Tumor Heterogeneity Diminishes Immune Response in Melanoma. Cell. 2019;179(1):219 - 35.e21.

15. Huang W, Zhou T, Ma L, Sun H, Gong H, Wang J, et al. Standard uptake value and metabolic tumor volume of $1 \hat{a} \bigotimes, F-F D G$ PET/CT predict short-term outcome early in the course of chemoradiotherapy in advanced non-small cell lung cancer. Eur J Nucl Med Mol Imaging. 2011;38(9):1628-35.

16. Herrmann K, Buck AK, Schuster T, Junger A, Wieder HA, Graf N, et al. Predictive value of initial 18FFLT uptake in patients with aggressive non-Hodgkin lymphoma receiving R-CHOP treatment. J Nucl Med. 2011;52(5):690-6.

17. Lehtiö K, Eskola O, Viljanen T, Oikonen V, Grönroos T, Sillanmäki L, et al. Imaging perfusion and hypoxia with PET to predict radiotherapy response in head-and-neck cancer. Int J Radiat Oncol Biol Phys. 2004;59(4):971-82.

18. Askoxylakis V, Dinkel J, Eichinger M, Stieltjes B, Sommer G, Strauss LG, et al. Multimodal hypoxia imaging and intensity modulated radiation therapy for unresectable non-small-cell lung cancer: the HIL trial. Radiat Oncol. 2012;7:157.

19. Luan X, Huang Y, Gao S, Sun X, Wang S, Ma L, et al. 18F-alfatide PET/CT may predict short-term outcome of concurrent chemoradiotherapy in patients with advanced non-small cell lung cancer. Eur J Nucl Med Mol Imaging. 2016;43(13):2336-42.

20. Zhang H, Liu N, Gao S, Hu X, Zhao W, Tao R, et al. Can an 'â囚,F-ALF-NOTA-PRGD2 PET/CT Scan Predict Treatment Sensitivity to Concurrent Chemoradiotherapy in Patients with Newly Diagnosed Glioblastoma. J Nucl Med. 2016;57(4):524-9.

21. Li L, Ma L, Shang D, Liu Z, Yu Q, Wang S, et al. Pretreatment PET/CT imaging of angiogenesis based on 18F-RGD tracer uptake may predict antiangiogenic response. Eur $\mathrm{J}$ Nucl Med Mol Imaging. 2019;46(4):940-7. 
22. Ferrara N, Kerbel RS. Angiogenesis as a therapeutic target. Nature. 2005;438(7070):967-74.

23. Kalluri R. Basement membranes: structure, assembly and role in tumour angiogenesis. Nat Rev Cancer. 2003;3(6):422-33.

24. Rosenberg RD. Vascular-bed-specific hemostasis and hypercoagulable states: clinical utility of activation peptide assays in predicting thrombotic events in different clinical populations. Thromb Haemost. 2001;86(1):41-50.

25. Rosenberg RD, Aird WC. Vascular-bed-specific hemostasis and hypercoagulable states. N Engl J Med. 1999;340(20):1555-64.

26. Jain RK. Normalization of tumor vasculature: an emerging concept in antiangiogenic therapy. Science. 2005;307(5706):58-62.

27. Meert AP, Paesmans M, Martin B, Delmotte P, Berghmans T, Verdebout JM, et al. The role of microvessel density on the survival of patients with lung cancer: a systematic review of the literature with meta-analysis. Br J Cancer. 2002;87(7):694-701.

28. Jain RK. Normalizing tumor microenvironment to treat cancer: bench to bedside to biomarkers. J Clin Oncol. 2013;31(17):2205-18.

29. Jain RK. Antiangiogenesis strategies revisited: from starving tumors to alleviating hypoxia. Cancer Cell. 2014;26(5):605-22.

30. Beer AJ, Lorenzen S, Metz S, Herrmann K, Watzlowik P, Wester HJ, et al. Comparison of integrin alphaVbeta3 expression and glucose metabolism in primary and metastatic lesions in cancer patients: a PET study using 18F-galacto-RGD and 18F-FDG. J Nucl Med. 2008;49(1):22-9.

31. Beer AJ, Haubner R, Sarbia M, Goebel M, Luderschmidt S, Grosu AL, et al. Positron emission tomography using [18F]Galacto-RGD identifies the level of integrin alpha(v)beta3 expression in man. Clin Cancer Res. 2006;12(13):3942-9.

\section{Tables}




\section{Table 1}

Clinicopathological features of the patients with locally advanced NSCLC

\begin{tabular}{|c|c|}
\hline Characteristics & Number of cases (\%) \\
\hline Age & $60 \pm 9.31$ \\
\hline$>60$ & $14 \llbracket 50.00 \rrbracket$ \\
\hline$\leq 60$ & $14 \llbracket 50.00 \rrbracket$ \\
\hline \multicolumn{2}{|l|}{ Gender } \\
\hline Male & $24 \llbracket 85.71 \rrbracket$ \\
\hline Female & $4 \bigotimes 14.29 \rrbracket$ \\
\hline \multicolumn{2}{|l|}{ Stage } \\
\hline$\triangle A$ & $13 \rrbracket 46.43 \rrbracket$ \\
\hline$\triangle \mathrm{B}$ & 15囚53.57ه \\
\hline \multicolumn{2}{|l|}{ Pathological type } \\
\hline Adenocarcinoma & $11 \rrbracket 39.29 \rrbracket$ \\
\hline Squamous cell carcinoma & $17 \rrbracket 60.71 \rrbracket$ \\
\hline \multicolumn{2}{|l|}{ Short-term outcome (RECIST) } \\
\hline Complete response & $1 \rrbracket 3.57 \rrbracket$ \\
\hline Partial response & $14 \llbracket 50.00 \rrbracket$ \\
\hline Stable disease & 8ه28.57ه \\
\hline Progressive disease & $5 \rrbracket 17.86 \rrbracket$ \\
\hline PFS & 17 (range 7.89 26.12) months \\
\hline OS & 36 (range 11.24 60.76) months \\
\hline 1 year survival rate & $25(89.29)$ \\
\hline 3 years survival rate & $15(53.57)$ \\
\hline 5 years survival rate & $6(21.43)$ \\
\hline
\end{tabular}


Table 2

Results of correlation and regression analysis between variables with PFS and OS

\begin{tabular}{|c|c|c|c|c|c|c|c|c|}
\hline & \multicolumn{4}{|l|}{ PFS $^{a}$} & \multicolumn{4}{|l|}{$O S^{b}$} \\
\hline & \multirow[t]{2}{*}{$\mathrm{t}$} & \multirow[t]{2}{*}{ Sig. } & \multicolumn{2}{|c|}{$95.0 \% \mathrm{Cl}$} & \multirow[t]{2}{*}{$\mathrm{t}$} & \multirow[t]{2}{*}{ Sig. } & \multicolumn{2}{|c|}{$95.0 \% \mathrm{Cl}$} \\
\hline & & & $\begin{array}{l}\text { Lower } \\
\text { bound }\end{array}$ & $\begin{array}{l}\text { Upeper } \\
\text { bound }\end{array}$ & & & $\begin{array}{l}\text { Lower } \\
\text { bound }\end{array}$ & $\begin{array}{l}\text { Upeper } \\
\text { bound }\end{array}$ \\
\hline $\operatorname{SUV}_{P}$ & -3.802 & 0.001 & -25.779 & -7.582 & -3.145 & 0.005 & -22.410 & -4.601 \\
\hline$S_{U V} V_{L N}$ & 3.509 & 0.002 & 8.531 & 33.196 & 1.662 & 0.111 & -2.395 & 21.745 \\
\hline $\mathrm{TBR}_{\mathrm{P}}$ & 3.239 & 0.004 & 3.874 & 17.662 & 1.641 & 0.115 & -1.409 & 12.086 \\
\hline $\mathrm{TBR}_{\mathrm{LN}}$ & -3.809 & 0.001 & -30.401 & -8.967 & -1.783 & 0.088 & -19.504 & 1.473 \\
\hline T/LN & -3.023 & 0.006 & -27.602 & -5.140 & -1.465 & 0.157 & -18.756 & 3.228 \\
\hline
\end{tabular}

a. Dependent variable, PFS; b. Dependent variable, OS

\section{Table 3}

Parameters of pretreatment for ${ }^{18} \mathrm{~F}$-Alfatide $\otimes \mathrm{PET} / \mathrm{CT}$ scans

\begin{tabular}{lllll}
\hline Parameters & All patients & Responders & Non-responders & $P$ value \\
\hline SUV $_{\mathrm{P}}{ }^{*}$ & $5.51 \pm 2.50$ & $4.61 \pm 1.94$ & $6.55 \pm 2.74$ & 0.039 \\
\hline $\mathrm{SUV}_{\mathrm{LN}}$ & $3.47 \pm 1.71$ & $3.59 \pm 1.81$ & $3.33 \pm 1.65$ & 0.699 \\
\hline $\mathrm{T} / \mathrm{LN}$ & $2.04 \pm 2.30$ & $1.42 \pm 0.53$ & $2.75 \pm 3.25$ & 0.172 \\
\hline $\mathrm{TBR}_{\mathrm{P}}{ }^{*}$ & $7.73 \pm 6.09$ & $5.34 \pm 3.03$ & $10.49 \pm 7.58$ & 0.023 \\
\hline $\mathrm{TBR}_{\mathrm{LN}}$ & $4.43 \pm 2.31$ & $4.20 \pm 2.53$ & $4.68 \pm 2.11$ & 0.589 \\
\hline *. $P<0.05$ & & & &
\end{tabular}




\section{Table 4}

Area under the curve of $S_{U} V_{P}, S U V_{L N}, T / L N, T B R_{P}$ and $T B R_{L N}$ for predicting tumor response Variables Area $\quad \mathrm{SE}^{\mathrm{a}} \quad$ Asymptotic Sig. ${ }^{\mathrm{b}} \quad$ Asymptotic $95 \%$ confidence interval

\begin{tabular}{llllll} 
& & & & Lower bound & Upeper bound \\
SUV $_{\mathrm{P}}$ & 0.703 & 0.105 & 0.069 & 0.497 & 0.908 \\
\hline SUV $_{\mathrm{LN}}$ & 0.477 & 0.114 & 0.836 & 0.254 & 0.700 \\
\hline T/LN & 0.715 & 0.098 & 0.053 & 0.523 & 0.907 \\
\hline TBR $_{\mathrm{P}}{ }^{*}$ & 0.764 & 0.091 & 0.018 & 0.586 & 0.943 \\
\hline TBR $_{\mathrm{LN}}$ & 0.585 & 0.111 & 0.447 & 0.367 & 0.803
\end{tabular}

a. Under the nonparametric assumption

b. Null hypothesis: true area $=0.5$

*. $P<0.05$

Table 5

Area under the curve of $S_{U} V_{P}, S U V_{L N}, T / L N, T B R_{P}$ and $T B R_{L N}$ for predicting Survival Variables Area SE ${ }^{\mathrm{a}} \quad$ Asymptotic Sig. ${ }^{\mathrm{b}} \quad$ Asymptotic $95 \%$ confidence interval Lower bound Upeper bound

\begin{tabular}{lccccc} 
SUV $_{\mathrm{P}}{ }^{*}$ & 0.942 & 0.042 & 0.000 & 0.860 & 1.000 \\
\hline SUV $_{\mathrm{LN}}$ & 0.719 & 0.099 & 0.065 & 0.524 & 0.914 \\
\hline $\mathrm{T} / \mathrm{LN}$ & 0.731 & 0.109 & 0.052 & 0.518 & 0.944 \\
$\mathrm{TBR}_{\mathrm{P}}{ }^{*}$ & 0.895 & 0.060 & 0.001 & 0.778 & 1.000 \\
$\mathrm{TBR}_{\mathrm{LN}}$ & 0.678 & 0.118 & 0.134 & 0.447 & 0.910
\end{tabular}

a. Under the nonparametric assumption

b. Null hypothesis: true area $=0.5$

*. $P<0.05$ 


\section{Table 6}

The specificity, sensitivity, and accuracy of $S U V_{P}, S U V_{L N}, T / L N, T B R_{P}$ and $T B R_{L N}$ for predicting survival

\begin{tabular}{lllll} 
Parameters & Threshold & Sensitivity (\%) & Specificity (\%) & Accuracy (\%) \\
\hline SUV $_{P}$ & 4.64 & 84.21 & 100.00 & 89.29 \\
\hline SUV $_{\text {LN }}$ & 3.43 & 92.31 & 53.33 & 71.43 \\
\hline T/LN & 1.73 & 92.31 & 53.33 & 71.43 \\
\hline TBR $_{P}$ & 4.92 & 93.75 & 66.67 & 82.14 \\
\hline TBR $_{\text {LN }}$ & 3.68 & 86.67 & 53.85 & 71.43
\end{tabular}

\section{Figures}

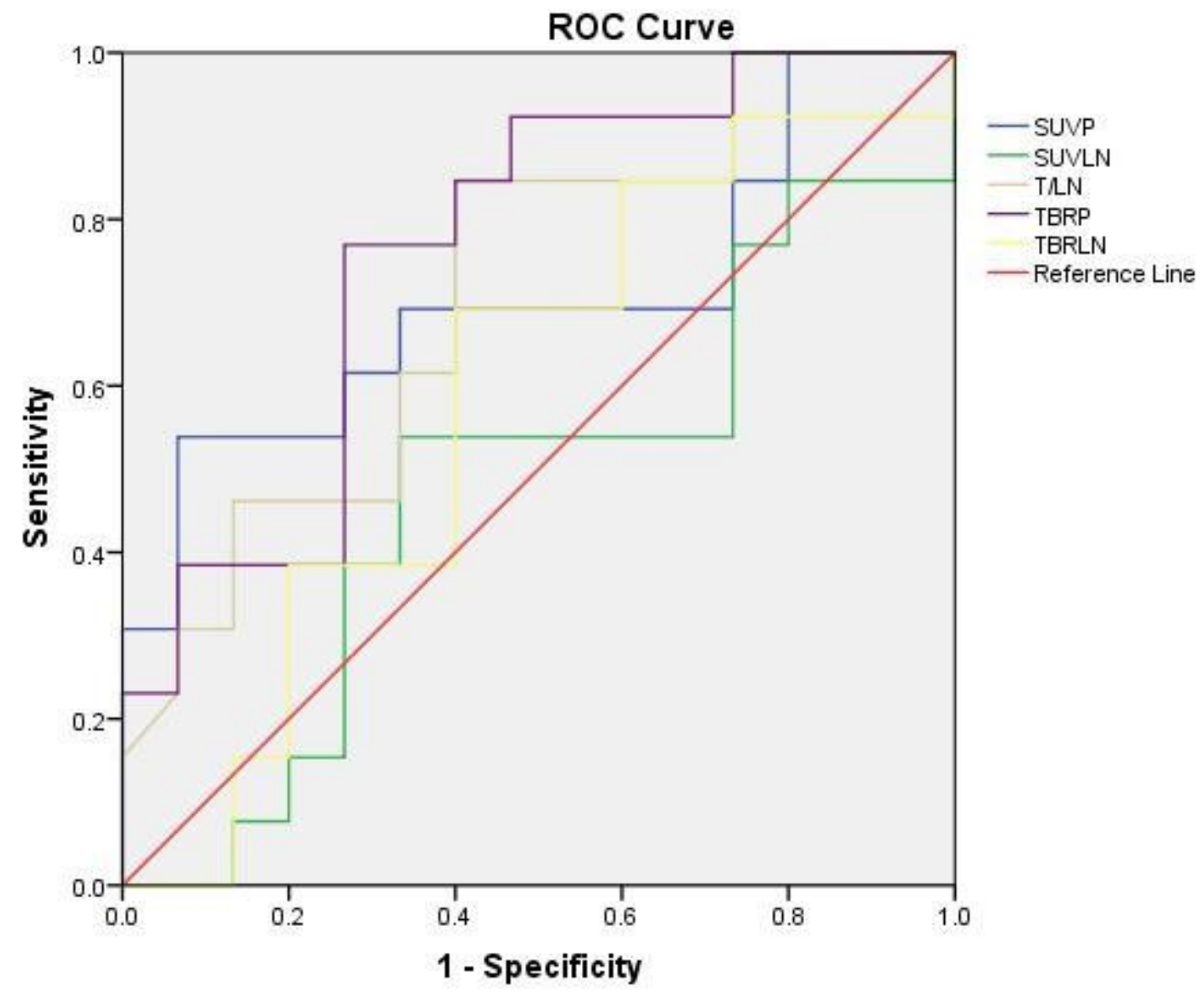

Figure 1 
ROC curve analysis of the predictive value of SUVs on baseline 18F-Alfatide $\triangle$ PET/CT imaging for shortterm response to CCRT, revealing a significant AUC of 0.764 ( $P=0.018$ ) for TBRP (cutoff value, 6.52).

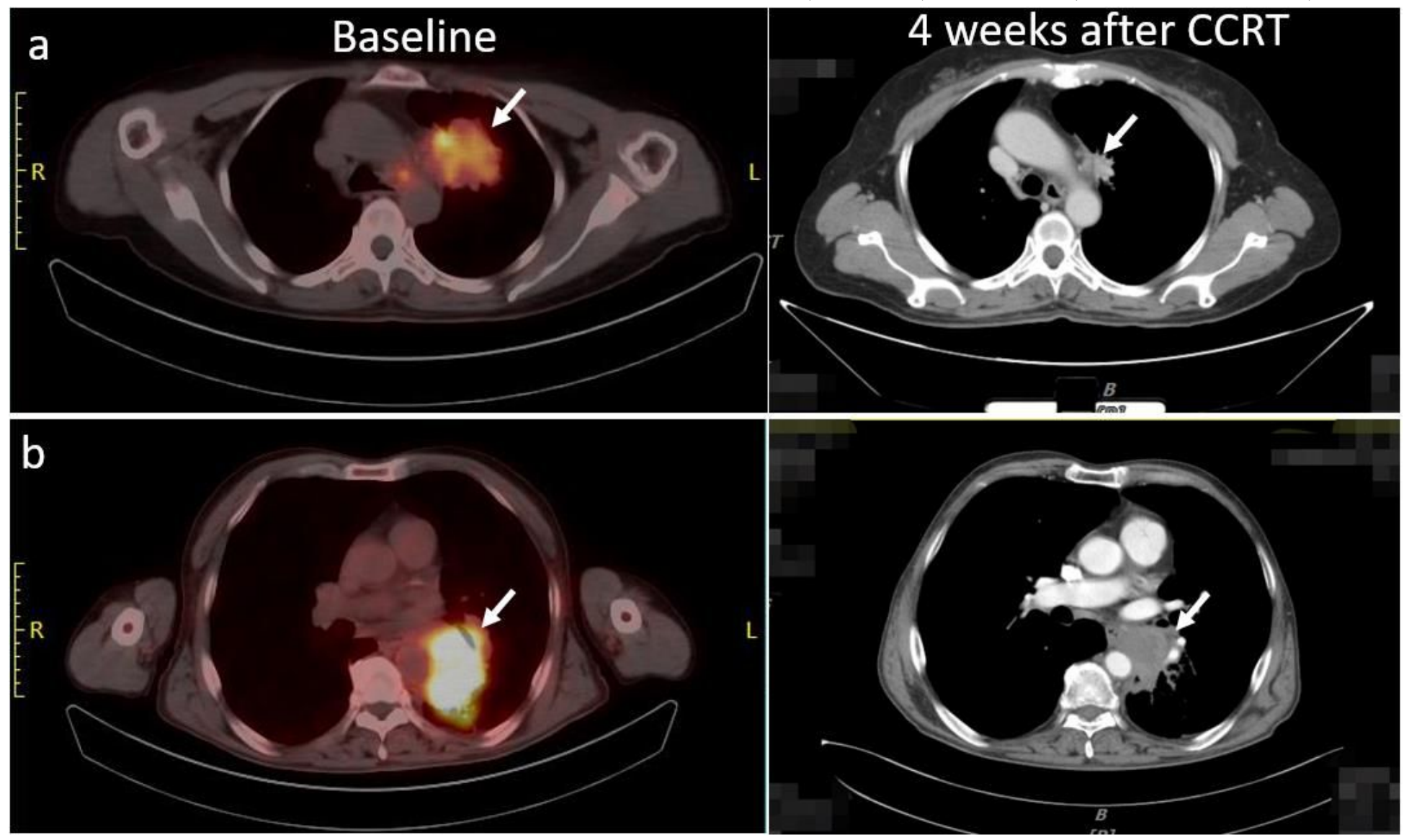

Figure 2

Two representative 18F-Alfatide $\otimes$ PET/CT scans of responding tumors (a: SUVmax=5.18) and nonresponding tumors (b: SUVmax=9.80). (Top panel) Scans from a patient with 18F-Alfatide uptake at baseline and 4 weeks after CCRT who showed a partial response to CCRT, and (bottom panel) scans from another patient with stable disease. 


\section{ROC Curve}

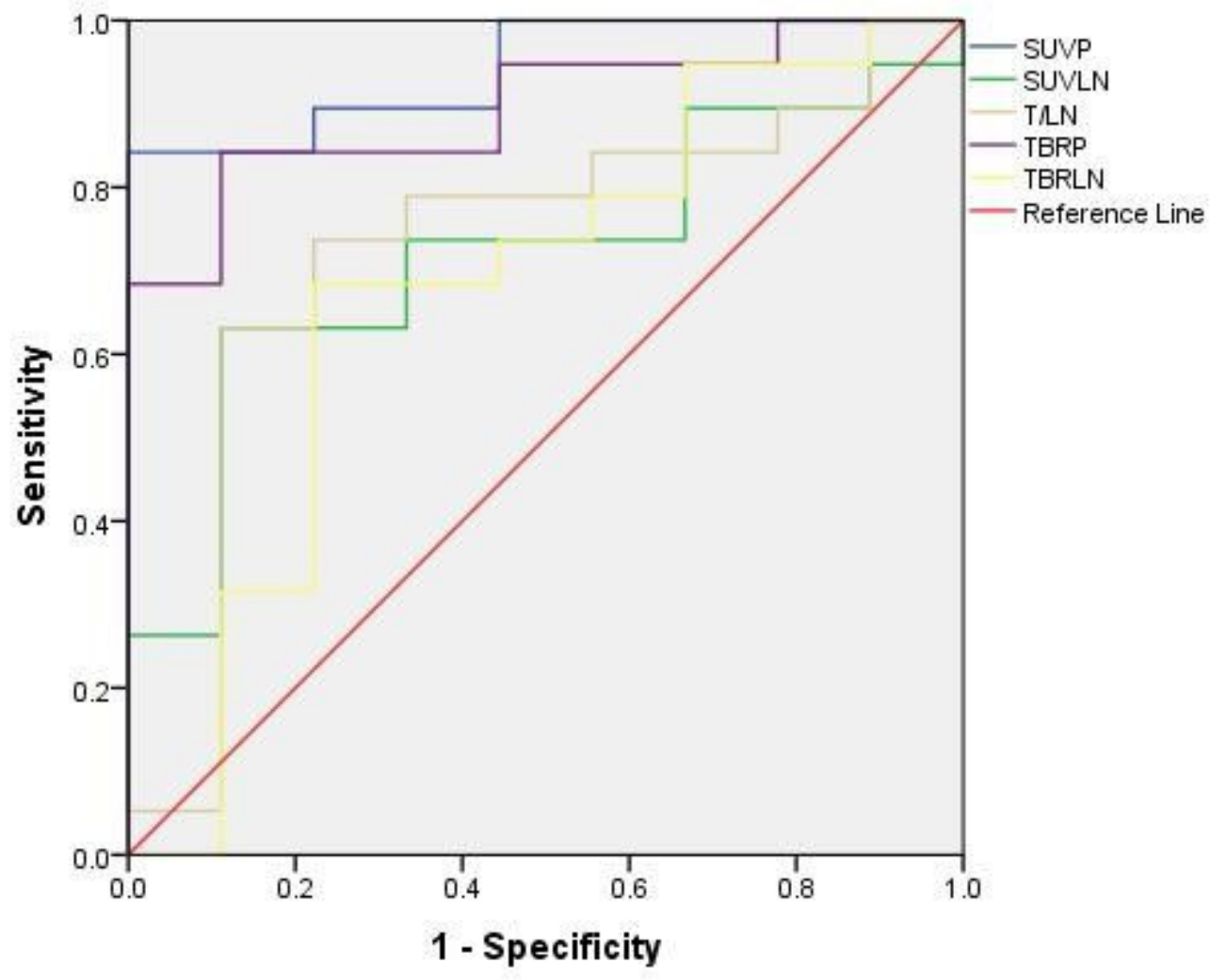

Figure 3

ROC curve analysis of the predictive value of SUVs on baseline 18F-Alfatide $\otimes$ PET/CT imaging, applying OS as the dichotomous characteristic, revealing significant AUC values of $0.942(P<0.001)$ for SUVP (cutoff value, 4.64) and 0.895 ( $P=0.001)$ for TBRP (cutoff value, 4.92). 